\title{
Photoinduced Diamagnetic to Paramagnetic Phase Transition in Organic Radical Crystals Studied by Microscopic IR Measurements
}

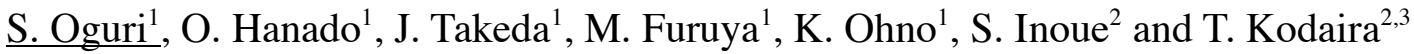 \\ ${ }^{1}$ Department of Physics, Graduate School of Engineering, Yokohama National University \\ Yokohama 240-8501, Japan \\ ${ }^{2}$ National Institute of Advanced Industrial Science and Technology (AIST) \\ Tukuba 305-8565, Japan \\ ${ }^{3}$ PRESTO, Japan Science and Technology Corporation, Japan
}

\begin{abstract}
Photoinduced magnetic phase transition in an organic radical 1,3,5-trithia-2,4,6triazapentalenyl (TTTA) crystal has been studied by microscopic IR measurements. Both photoexcitations of the charge-transfer and intramolecular $\pi-\pi$ transition bands induce a low-temperature diamagnetic to high-temperature paramagnetic phase transition but with different threshold photon densities. The behavior of the photoinduced magnetic phase transition in TTTA is discussed in comparison with previous results measured by the photoinduced Raman and ESR measurements.
\end{abstract}

PACS: $78.30 . \mathrm{Jw}, 75.30 . \mathrm{Kz}$

Keywords: Photoinduced magnetic phase transition, Mott insulator, radical, TTTA, IR

Corresponding author:

Jun Takeda

Yokohama National University

79-5 Tokiwadai, Hodogaya, Yokohama 240-8501, Japan

Tel: +81-45-339-3953

Fax: +81-45-339-3953

E-mail: jun@ynu.ac.jp 


\section{Introduction}

Structural phase transitions in low-dimensional organic materials are of great current interest. Recently photoinduced phase transition (PIPT) phenomena have been extensively studied in several low-dimensional organic materials such as $\pi$ conjugated polymers [1,2] and charge-transfer (CT) complexes [3-5]. In PIPT phenomena, light irradiation stimulates a macroscopic phase transition, whereby cooperative interactions of photoexcited states are thought to play an important role on PIPT, leading to nonlinear properties such as existence of a threshold photon density for the occurrence of PIPT. Because magnetic and/or chromic properties of materials can be controlled by light irradiation, investigation of PIPT phenomena is valuable not only for its fundamental interest but also for application of molecularbased optical devices.

Since a large first order magnetic phase transition with a wide thermal hysteresis loop over wide temperature range was found in 1,3,5-trithia-2,4,6-triazapentalenyl (TTTA) crystal [6], an organic radical crystal is expected to be a new type of materials suitable for studying PIPT phenomena. In this material, the high temperature $(\mathrm{HT})$ phase is paramagnetic due to a uniform one-dimensional stacking of the radical molecules, while the low temperature (LT) phase is diamagnetic due to strong dimerization along the stacking direction. The electronic structure of TTTA crystal has been calculated theoretically, and the HT phase is shown to be a simplest molecular Mott insulator among the molecular-based materials ever found $[7,8]$.

Since TTTA shows bistability over a wide temperature range near room temperature, the PIPT has been examined through photoinduced Raman and ESR measurements [9-11]. The photoinduced diamagnetic to paramagnetic phase transition in TTTA is clearly observed with a threshold photon density. In the previous Raman measurements, however, the longtime laser irradiation by an excitation laser source made TTTA crystals degraded/damaged. Therefore photoinduced IR measurement seems to be a better spectroscopic tool to obtain elaborate experimental results on PIPT phenomena of TTTA. In this paper, we report on PIPT phenomena in an organic radical TTTA crystal studied through photoinduced microscopic IR measurement. The observed experimental results show 
qualitatively the same but quantitatively different behavior in comparison with those in previous work [10].

\section{Experimental}

TTTA was synthesized by the method previously reported [12]. The single crystals of TTTA were grown by a vacuum sublimation method. By controlling crystal growth temperature, we could selectively obtain either the HT or LT phase crystal [13]. The typical size of TTTA single crystal is $0.5 \times 0.3 \times 0.2 \mathrm{~mm}^{3}$. In order to measure IR transmission spectra, we also prepared thinner TTTA samples coated on $\mathrm{KBr}$ substrate. Since TTTA distributes island-likely on the substrate, samples obtained are polycrystalline. The average thickness of the thinner samples is $\sim 20 \mu \mathrm{m}$.

The IR reflection and transmission spectra of TTTA were measured by a microscopic FT-IR spectrometer with a spectral resolution of $1-4 \mathrm{~cm}^{-1}$ at room temperature. The laser irradiation upon sample to drive PIPT was performed by second $(532 \mathrm{~nm}, 2.33 \mathrm{eV})$ and third $(355 \mathrm{~nm}, 3.49 \mathrm{eV})$ harmonics of a pulsed Nd:YAG laser, whose excitation wavelengths locate at the CT and intramolecular $\pi$ $\pi$ transition bands, respectively [14].

\section{Experimental Results and Discussion}

Figure 1 shows polarized IR reflection spectra of the HT (a) and LT (b) phase crystals between 650 and $850 \mathrm{~cm}^{-1}$ at room temperature. The IR reflection spectra of the HT phase in this frequency region consists of mainly 3 vibrational modes with wavenumbers of 681, 774 and $832 \mathrm{~cm}^{-1}$ (downward arrows), which are strongly polarized parallel to the molecular stacking direction. In the LT phase, these modes are slightly shifted and the $681 \mathrm{~cm}^{-1}$ mode becomes doublet $\left(672\right.$ and $\left.681 \mathrm{~cm}^{-1}\right)$ as shown by open circles. This doublet structure is observed when polarization of light is parallel to the stacking direction. Since the LT phase shows strong dimerization along the stacking direction, periodicity along the stacking direction in the LT phase 
relative to that in the HT phase is expected to be twice. Therefore the observed doublet structure in the LT phase comes from the zone folding effect and clearly indicates that the magnetic phase transition of TTTA is accompanied with strong dimerization.

To investigate the photoinduced magnetic phase transition in TTTA, we measured photoinduced IR transmission spectra for the LT phase polycrystalline samples coated on $\mathrm{KBr}$ substrate after a single-shot laser irradiation with different photon densities. Figure 2 shows the IR transmission spectra between 650 and 900 $\mathrm{cm}^{-1}$ under the photoexcitation of the CT band at room temperature. The vertical bars indicate the characteristic vibrational modes of the LT phase. With increasing the irradiated photon density, the shape of the IR transmission spectrum becomes close to that of the HT phase, indicating that the LT phase transforms to the HT phase by the photoexcitation. The amount of the HT phase photogenerated in the LT phase is evaluated by the same procedure as previously reported [10].

Figure 3 shows conversion rate from the LT to HT phase as a function of irradiated photon density with different photoexcitation wavelengths. The solid lines are visual guides for eyes. The conversion takes place with a clear threshold photon density whose values lie at $\sim 7 \times 10^{19} / \mathrm{cm}^{3}$ and $\sim 7 \times 10^{18} / \mathrm{cm}^{3}$ for the photoexcitation of the CT and intramolecular $\pi-\pi$ transition bands, respectively. This behavior clearly shows that the nonlinear process related to PIPT takes place in TTTA.

Our experimental results show that both the photoexcitations of the CT and intramolecular $\pi-\pi$ transition bands can induce the diamagnetic to paramagnetic phase transition but with different threshold photon densities. Since the photoexcitation of the intramolecular $\pi-\pi$ transition band has much excess energy, photogenerated HT fractions are quite mobile and then might coalesce into a larger unit through mutual encounter to be more stable. This may create a larger size of the HT fraction in the LT phase crystal as the precursor that becomes a trigger of PIPT, resulting in the occurrence of the PIPT with smaller threshold photon density.

The same qualitative behavior was already reported through photoinduced Raman scattering measurements [10], but the observed threshold photon densities are one order of magnitude larger than those obtained in the present work. In the photoinduced Raman scattering measurements, TTTA single crystals were used. On 
the other hand, polycrystalline TTTA samples coated on KBr substrate, which consist of island-like small size grains, are used in this experiment. The observed different threshold photon densities between the two experiments might come from difference of the sample morphology. Further elaborate experiments using TTTA samples with different kinds of sizes and shapes are needed to elucidate the origin of the above difference.

In conclusion, we observed the photoinduced magnetic phase transition in TTTA through photoinduced microscopic IR measurements. Both photoexcitations of the $\mathrm{CT}$ and intramolecular $\pi-\pi$ transition bands induce the LT (diamagnetic) to the HT (paramagnetic) phase transition but with different threshold photon densities. These results indicate that the PIPT takes place in an organic radical crystal TTTA and the magnetic and chromic properties of TTTA can be optically controlled. The observed smaller threshold photon densities compared to those obtained from the previous photoinduced Raman scattering measurements might come from the difference of the sample morphology.

\section{Acknowledgements}

This work was supported in part by Grant-in-Aid for Scientific Research B (No. 15340094) from Japan Society for the Promotion of Science (JSPS). The financial support of the Yokohama Academic Foundation to S. O. is gratefully acknowledged. 
References

[1] S. Koshihara, Y. Tokura, K. Takeda, T. Koda, Phys. Rev. Lett. 68 (1992) 1148.

[2] N. Hosaka, H. Tachibana, N. Shiga, M. Matsumoto Y. Tokura, Phys. Rev. Lett. 82 (1999) 1672.

[3] S. Koshihara, Y. Tokura, T. Mitani, G. Saito, T. Koda, Phys. Rev. B 42, 6853 (1990).

[4] T. Suzuki, T. Sakamaki, K. Tanimura, S. Koshihara, Y. Tokura, Phys. Rev. B 60 (1999) 6191.

[5] K. Tanimura, I. Akimoto, Phase Transitions 75 (2002) 715.

[6] W. Fujita, K. Awaga, Science 286 (1999) 261.

[7] M. Furuya, K. Ohno, T. Morisato, Y. Kawazoe, J. Takeda, Trans. Mater. Res.Soc. Jpn. 28 (2003) 911.

[8] M. Furuya, J. Takeda, S. Ishii, K. Ohno, in preparation.

[9] J. Takeda, M. Imae, S. Kurita, T. Kodaira, Phase Transitions 75 (2002) 863.

[10] J. Takeda, M. Imae, O. Hanado, S. Kurita, M. Furuya, K. Ohno, T. Kodaira, Chem. Phys. Lett. 378 (2003) 456.

[11] H. Matsuzaki, W. Awaga, H. Okamoto, Phys. Rev. Lett. 91 (2003) 17403.

[12] G. Wolmershuäuser, R. Johann, Angew. Chem. Int. Engl. 28 (1989) 920.

[13] unpublished.

[14] W. Fujita, K. Awaga, H. Matsuzaki, H. Okamoto, Phys. Rev. B 65 (2002) 064434. 


\section{Figure Captions}

Figure 1

Polarized IR reflection spectra of the HT (a) and LT (b) phases at room temperature.

Figure 2

Photoinduced IR transmission spectra under the photoexcitation of the CT band at room temperature.

Figure 3

Conversion rate from the LT to HT phase as a function of irradiated photon density with different photoexcitation wavelengths. 


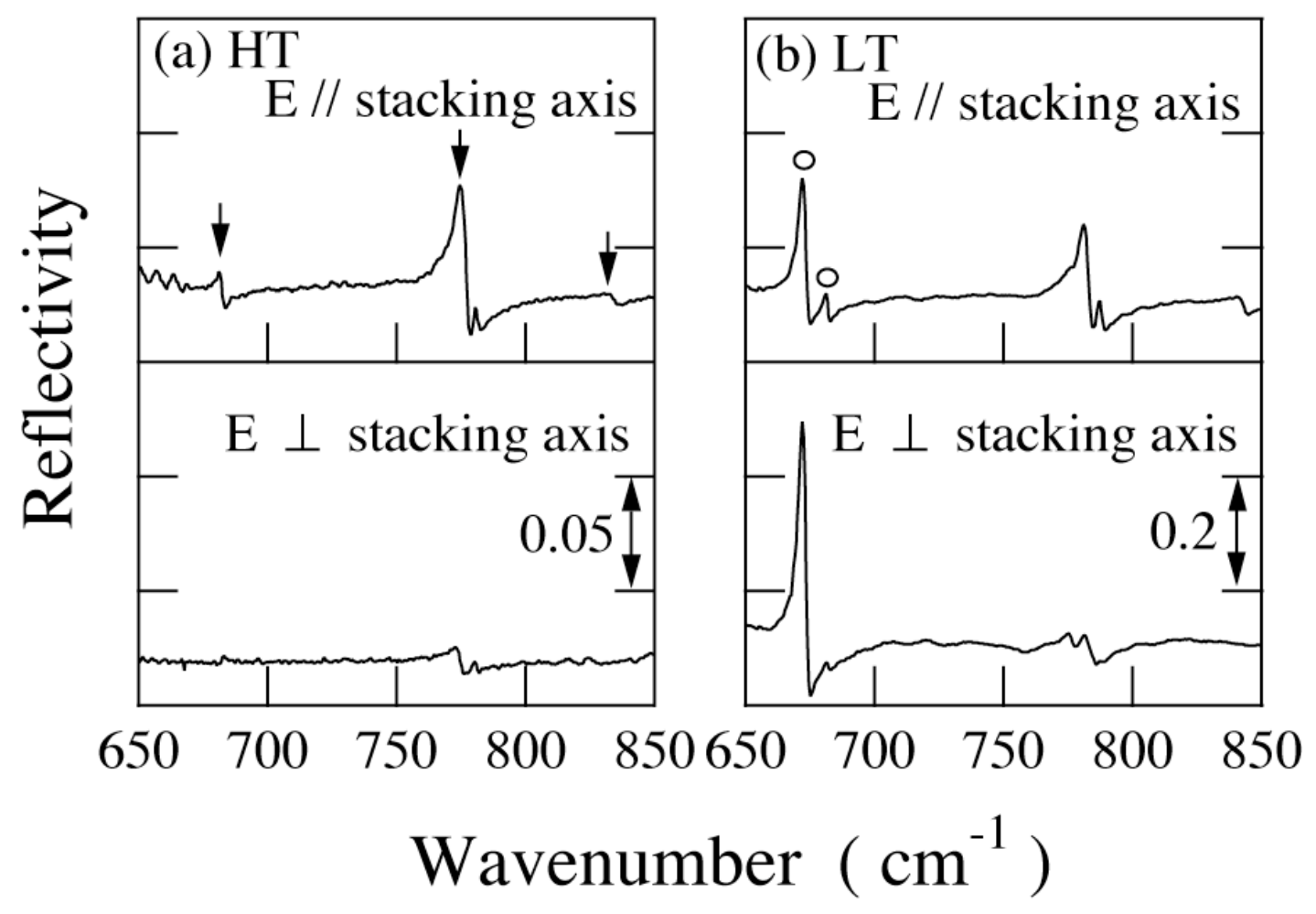

Fig. 1 S. Oguri et al. 


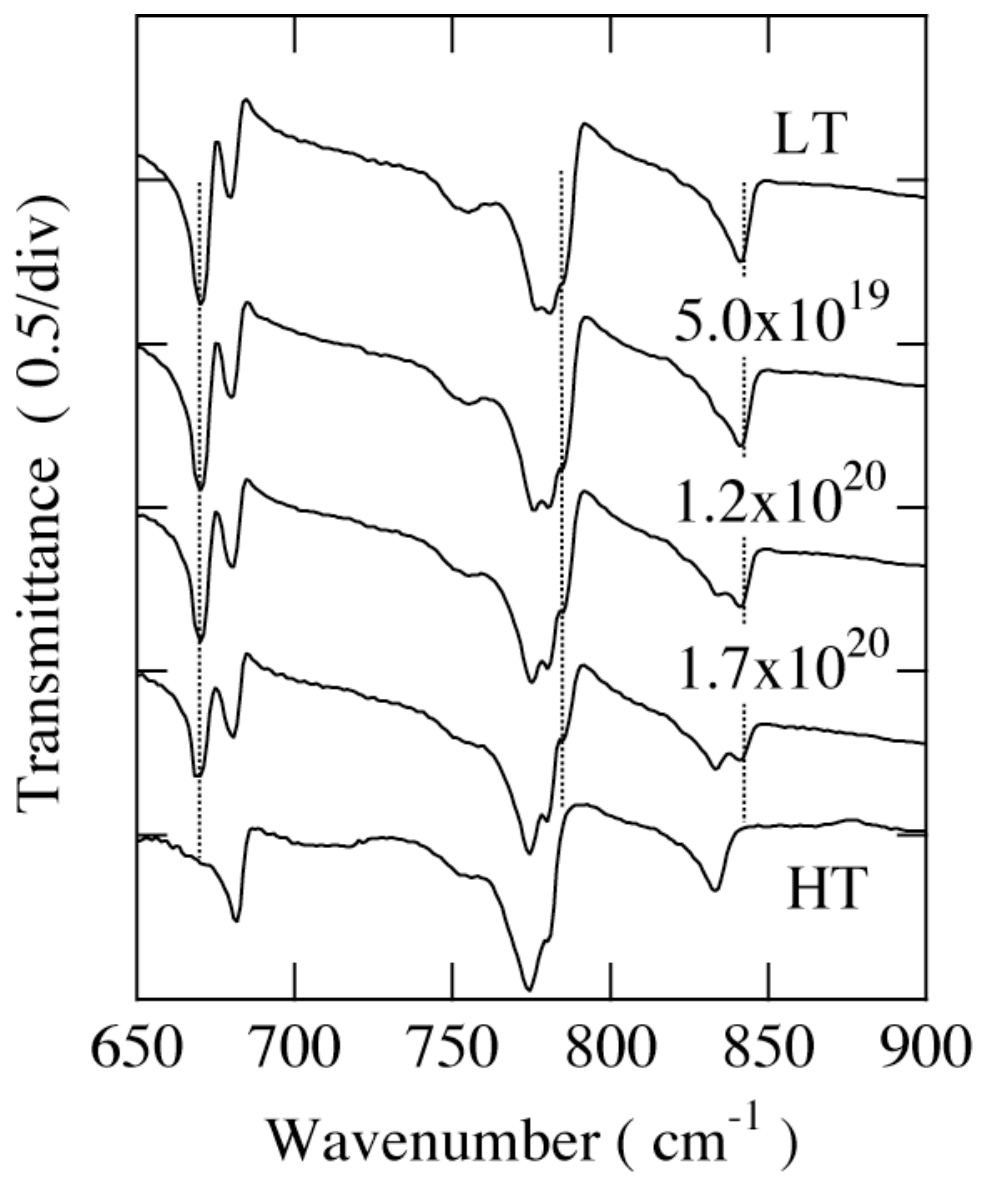

Fig. 2 S. Oguri et al. 


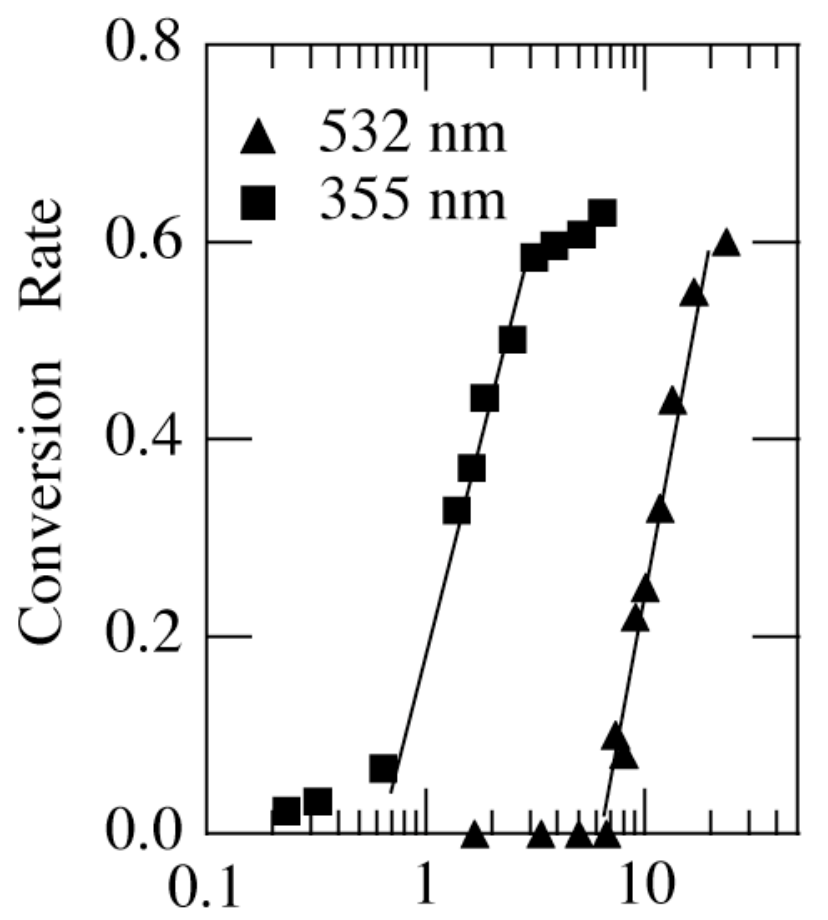

Photon Density ( $\times 10^{19} / \mathrm{cm}^{3}$ )

Fig. 3 S. Oguri et al. 